Received: 07.12 .2020

Revised: 17.01 .2021

Accepted: 26.02 .2021

DOI: $10.17804 / 2410-9908.2021 .1 .038-050$

\title{
EVOLUTION OF THE STRUCTURE OF ANNEALED HAFNIUM BRONZE NANOSTRUCTURED BY HIGH PRESSURE TORSION
}

\author{
A. V. Stolbovsky ${ }^{\text {a)* }}$, V. V. Popov ${ }^{\text {b) }}$, R. M. Falahutdinov ${ }^{\text {c) }}$, and S. A. Murzinova ${ }^{\text {d) }}$ \\ M.N. Miheev Institute of Metal Physics, Ural Branch of the Russian Academy of Sciences, \\ 18 S. Kovalevskoy St., Ekaterinburg, 620108, Russian Federation \\ a) iD http://orcid.org/0000-0001-5228-7857 stolbovsky@imp.uran.ru; \\ b) (iD http://orcid.org/0000-0002-9291-0102 vpopov@imp.uran.ru; \\ c) (iD http://orcid.org/0000-0001-8505-9455 falahutdinov@imp.uran.ru; \\ d) (iD http://orcid.org/0000-0001-5240-2966 ख murzinova@imp.uran.ru \\ *Corresponding author. E-mail: stolbovsky@imp.uran.ru \\ Address for correspondence: ul. S. Kovalevskoy, 18, Ekaterinburg, 620108, Russian Federation \\ Tel.: +7 34337835 74; fax: +7 3433745244
}

The effect of severe plastic deformation by 1, 3 and 5 revolutions of high pressure torsion (HPT) on the structure and mechanical properties of low-alloyed hafnium bronze $\mathrm{Cu}-0.78 \mathrm{wt} \% \mathrm{Hf}$ is studied. In the initial annealed state, hafnium is almost completely bonded into intermetallic compounds. It has been found that the structure of all the investigated bronze specimens subjected to HPT is stable and that it remains unchanged after unloading and prolonged ageing at room temperature. It is shown that all the specimens develop a dispersed submicrocrystalline structure gradient along the radius of the disk, with an average crystallite size of $200 \mathrm{~nm}$ after 1 revolution to $120 \mathrm{~nm}$ after 5 revolutions (at mid-radius). The structure is non-uniform even after 5 revolutions, this being confirmed by microhardness measurements. The high-pressure-torsion behavior of hafnium bronze with Hf bonded into precipitates has much in common with the behavior of pure copper. At the same time, in terms of the stability of the obtained structures at room temperature, the behavior of the alloy under study demonstrates much in common with that of low-alloyed tin bronze.

Keywords: severe plastic deformation, high-pressure torsion, nanostructuring, nanostructures, grain boundaries, hafnium bronze.

\section{Acknowledgments}

The electron microscopic study used the equipment of the Collaborative Access Center at the Testing Center of Nanotechnologies and Advanced Materials, IMP UB RAS.

The work was performed under the state assignment from FASO Russia (theme Function, registration number AAAA-A19-119012990095-0) and partially supported by the UB RAS Basic Research Program, project 18-10-2-37.

\section{References}

1. Valiev R.Z., Zhilyaev A.P., Langdon T.G. Bulk Nanostructured Materials: Fundamentals and Applications, New Jersey, TMS, Wiley, Hoboken, 2014, 440 p. ISBN 978-1-118-09540-9.

2. Gleiter H. Nanostructured materials: basic concepts and microstructure. Acta Mater., 2000, vol. 48, pp. 1-29. DOI: 10.1016/S1359-6454(99)00285-2. 
3. Wang Y.M., Chen M.W., Sheng H.W., Ma E. Nanocrystalline grain structures developed in commercial purity $\mathrm{Cu}$ by low temperature cold rolling. Mater. Res. Soc., 2002, vol. 17, no. 12, pp. 3004-3007. DOI: 10.1557/JMR.2002.0436.

4. Gertsman V.Y., Birringer R., Valiev R.Z., Gleiter H. On the structure and strength of ultrafine-grained copper produced by severe plastic deformation. Scr. Met. \& Mater., 1994., vol. 30, no. 2, pp. 229-234. DOI: 10.1016/0956-716X(94)90045-0.

5. Erbel S. Mechanical properties and structure of extremely strainhardened copper. Metals Technol., 1979, vol. 6, no. 1, pp. 482-486. DOI: 10.1179/030716979803276363.

6. Liao X.Z., Zhao Y.H., Zhu Y.T. Grain-size effect on the deformation mechanisms of nanostructured copper processed by high-pressure torsion. J. Appl. Phys., 2004, vol. 96, no. 1, pp. 636-640. DOI: 10.1063/1.1757035.

7. $\quad$ Dalla Torre F., Lapovok R., Sandlin J., Thomson P.F., Davies C.H.J., Pereloma E.V. Microstructures and properties of copper processed by equal channel angular extrusion for 1-16 passes. Acta Mater., 2004, vol. 52, no. 16, pp. 4819-4832. DOI: 10.1016/j.actamat.2004.06.040.

8. Valiev R.Z., Kozlov E.V., Ivanov Yu.F., Lian J., Nazarov A.A., Baudel B. Deformation behavior of ultra-fine-grained copper, Acta Met. \& Mater., 1994, vol. 42, no. 7, pp. 2467-2475. DOI: 10.1016/0956-7151(94)90326-3.

9. Alexandrov I.V., Dubravina A.A., Kim H.S. Nanostructure formation in copper subjected to high pressure torsion. Defect \& Diffus. Forum, 2002, vol. 208-209, pp. 229-232. DOI: 10.4028/www.scientific.net/DDF.208-209.229.

10. Brandstetter S., Zhang K., Escuadro A., Weertman Julia. Grain coarsening during compression of bulk nanocrystalline nickel and copper. Scr. Mater., 2008, vol. 58, no. 1, pp. 61-64. DOI: 10.1016/j.scriptamat.2007.08.042.

11. Horita Z., Langdon T.G. Microstructures and microhardness of an aluminum alloy and pure copper after processing by high-pressure torsion. Mater. Sci. \& Eng. A., 2005, vol. 410-411, pp. 422-425. DOI: 10.1016/j.scriptamat.2007.08.042.

12. Islamgaliev R.K., Chmelik F., Kuzel R. Thermal stability of submicron grained copper and nickel. Mater. Sci. \& Eng. A., 1997, vol. 237, no. 1, pp. 43-51. DOI: 10.1016/S09215093(97)00107-X.

13. Akhmadeev N.H., Kobelev N.P., Mulyukov R.R. The effect of heat treatment on the elastic and dissipative properties of copper with the submicrocrystalline structure. Acta Met. \& Mater., 1993, vol. 41, no. 4, pp. 1041-1046. DOI: 10.1016/0956-7151(93)90153-J.

14. Popov V.V., Stolbovskiy A.V., Popova E.N., Pilyugin V.P. Structure and Thermal Stability of $\mathrm{Cu}$ after Severe Plastic Deformation. Defect and Diffusion Forum, 2010, vol. 297-301, pp. 1312-1321. DOI: 10.4028/www.scientific.net/DDF.297-301.1312.

15. Stolbovsky A.V., Popov V.V., Popova E.N., Pilyugin V.P. Structure, thermal stability, and state of grain boundaries of copper subjected to high-pressure torsion at cryogenic temperatures. Bulletin of the Russian Academy of Sciences: Physics, 2014, vol. 78, pp. 908-916. DOI: $10.3103 / \mathrm{S} 1062873814090299$.

16. Stolbovsky A.V., Popov V.V., Popova E.N., Falakhutdinov R.M. Effect of severe plastic deformation by high-pressure torsion on the structure and properties of copper and tin bronze. Diagnostics, Resource and Mechanics of materials and structures, 2017, iss. 5, pp. 13-22. DOI: 10.17804/2410-9908.2017.5.013-022. Availabke at: http://dream-journal.org/issues/20175/2017-5_144.html (accessed: 19.02.2021)

17. Shangina D.V., Bochvar N.R., Dobatkin S.V. Structure and properties of ultrafine-grained $\mathrm{Cu}-\mathrm{Cr}$ alloys after high pressure torsion. Materials Science Forum, 2011, vol. 667-669, pp. 301-306. DOI: 10.4028/www.scientific.net/MSF.667-669.301.

18. Shangina D.V., Bochvar N.R., Dobatkin S.V. The effect of alloying with hafnium on the thermal stability of chromium bronze after severe plastic deformation. Journal of Materials Science, 2012, vol. 47, pp. 7764-7769. DOI: 10.1007/s10853-012-6525-9. 
19. Shangina D.V., Maksimenkova Yu.M., Bochvar N.R., Serebryany Vl., Raab G., Vinogradov A., Skrotzki Werner, Dobatkin S. Structure and properties of $\mathrm{Cu}$ alloys alloying with $\mathrm{Cr}$ and $\mathrm{Hf}$ after equal channel angular pressing. Advanced Materials Research, 2014, vol. 922, pp. 651-656. DOI: $10.4028 /$ www.scientific.net/AMR.922.651.

20. Dobatkin S.V., Shangina D.V., Bochvar N.R., Janečekc Miloš. Effect of deformation schedules and initial states on structure and properties of $\mathrm{Cu}-0.18 \% \mathrm{Zr}$ alloy after high-pressure torsion and heating. Materials Science and Engineering A, 2014, vol. 598, pp. 288-292. DOI: $10.1016 /$ j.msea.2013.12.104.

21. Shangina D.V., Gubicza J., Dodony E., Bochvar N.R., Straumal P.B., Tabachkova N.Yu. \& Dobatkin S.V. Improvement of strength and conductivity in $\mathrm{Cu}$-alloys with the application of highpressure torsion and subsequent heat-treatments. Journal of Materials Science, 2014, vol. 49, pp. 6674-6681. DOI: 10.1007/s10853-014-8339-4.

22. Dobatkin S.V., Gubicza J., Shangina D.V., Bochvara N.R., Tabachkova N.Y. High strength and good electrical conductivity in $\mathrm{Cu}-\mathrm{Cr}$ alloys processed by severe plastic deformation. Materials Letters, 2015, vol. 153, pp. 5-9. DOI: doi.org/10.1016/j.matlet.2015.03.144.

23. Dobatkin S.V., Bochvar N.R., Shangina D.V. Aging processes in ultrafine-grained lowalloyed bronzes subjected to equal channel angular pressing. Advanced Engineering Materials, 2015, vol. 17, pp. 1862-1868. DOI: 10.1002/adem.201500099.

24. Shangina D.V., Bochvar N.R., Gorshenkov M.V., Yanar H., Purcek G., Dobatkin S.V. Influence of microalloying with zirconium on the structure and properties of $\mathrm{Cu}-\mathrm{Cr}$ alloy after high pressure torsion. Materials Science and Engineering A, 2016, vol. 650, pp. 63-66. DOI: $10.1016 /$ j.msea.2015.10.008.

25. Purcek G., Yanar H., Demirtas M., Alemdag Y., Shangina D.V., Dobatkin S.V. Optimization of strength, ductility and electrical conductivity of $\mathrm{Cu}-\mathrm{Cr}-\mathrm{Zr}$ alloy by combining multi-route ECAP and aging. Materials Science and Engineering A., 2016, vol. 649, pp. 114-122. DOI: 10.1016/j.msea.2015.09.111.

26. Shangina D., Maksimenkova Yu., Bochvar N., Serebryany Vl., Raab Georgy, Vinogradov A., Skrotzki Werner \& Dobatkin S. Influence of alloying with hafnium on the microstructure, texture, and properties of $\mathrm{Cu}-\mathrm{Cr}$ alloy after equal channel angular pressing. Journal of Materials Science, 2016, vol. 51, pp. 5493- 5501. DOI: 10.1007/s10853-016-9854-2.

27. Stolbovsky A.V., Popov V.V., Popova E.N. Structure and Thermal Stability of Tin Bronze Nanostructured by High Pressure Torsion. Diagnostics, Resource and Mechanics of materials and structures, 2015, iss. 5, pp. 118-132. DOI: 10.17804/2410-9908.2015.5.118-132. Available at: http://dream-journal.org/issues/2015-5/2015-5_52.html (accessed: 27.06.2020).

28. Popov V.V., Popova E.N., Stolbovsky A.V., Falakhutdinov R.M. Evolution of the Structure of $\mathrm{Cu}-1 \% \mathrm{Sn}$ Bronze under High Pressure Torsion and Subsequent Annealing. Physics of Metals and Metallography, 2018, vol. 119, pp. 358-367. DOI: 10.1134/S0031918X18040154. 
Подана в журнал: 07.12.2020

УДК 669.35'6:539.89

DOI: $10.17804 / 2410-9908.2021 .1 .038-050$

\title{
ЭВОЛЮЦИЯ СТРУКТУРЫ ОТОЖЖЕННОЙ ГАФНИЕВОЙ БРОНЗЫ, НАНОСТРУКТУРИРОВАННОЙ КРУЧЕНИЕМ ПОД ВЫСОКИМ ДАВЛЕНИЕМ
}

\author{
А. В. Столбовский ${ }^{\mathrm{a}}{ }^{*}$, В. В. Попов ${ }^{6)}$, Р. М. Фалахутдинов ${ }^{\text {в) }}$, С. А. Мурзинова ${ }^{\text {r) }}$ \\ Институт физики металлов им. М.Н. Михеева УрО РАН, \\ д. 18, ул. С. Ковалевской, Россия, 620108, г. Екатеринбург, Российская Федераџия \\ (iD) \\ http://orcid.org/0000-0001-5228-7857 tolbovsky@imp.uran.ru; \\ (D) http://orcid.org/0000-0002-9291-0102 vopov@imp.uran.ru; \\ (iD http://orcid.org/0000-0001-8505-9455 図 falahutdinov@imp.uran.ru; \\ iD http://orcid.org/0000-0001-5240-2966 @ murzinova@imp.uran.ru \\ *Ответственный автор. Электронная почта: stolbovsky@imp.uran.ru \\ Адрес для переписки: ул. С. Ковалевской, 18, Екатеринбург, 620108, Российская Федерация \\ Тел.: +7 (343) 378-35-74; факс: (343) 374-52-44
}

Исследовано влияние интенсивной пластической деформации кручением под высоким давлением (КВД) на 1, 3 и 5 об. на структуру и механические свойства низколегированной гафниевой бронзы $\mathrm{Cu}-0,78$ мас. \% Hf. В исходном отожженном состоянии гафний практически полностью связан в интерметаллические выделения. Установлено, что структура всех исследованных образцов бронзы, подвергнутых КВД, стабильна и не претерпевает каких-либо изменений после снятия нагрузки и при длительном вылеживании. Показано, что во всех исследованных образцах формируется дисперсная субмикрокристаллическая структура, градиентная по радиусу диска, со средним размером кристаллитов от 200 нм после 1 об. до 120 нм после 5 об. (на середине радиуса). Даже после 5 об. структура является неоднородной, что подтверждается измерениями микротвердости. Поведение при кручении под высоким давлением гафниевой бронзы со связанным в выделения гафнием имеет много общего с поведением чистой меди. В то же время с точки зрения стабильности полученных структур при комнатной температуре поведение рассматриваемого сплава демонстрирует много общего с поведением, наблюдавшимся у низколегированной оловянистой бронзы.

Ключевые слова: интенсивная пластическая деформация, кручение под высоким давлением, наноструктурирование, наноструктуры, границы зерен, гафниевая бронза.

\section{1. Введение}

В современных условиях использование чистой меди в качестве конструкционного материала крайне ограничено из-за ее высокой пластичности в сочетании с относительно невысокой температурой плавления. При этом использование именно меди дает преимущество изделиям, для которых необходима высокая электро- или теплопроводность. Например, для деталей контактных групп в электротехнических изделиях или теплообменников, работающих при повышенных нагрузках, применение меди практически не имеет альтернатив.

На протяжении последних десятилетий велись постоянные поиски возможностей повысить прочностные характеристики меди для ее более широкого применения в качестве конструкционного материала с уникальными свойствами [1]. И только после появления нового подхода к получению материалов с высокой плотностью структурных дефектов [1-15] медь была предложена в качестве полноценного конструкционного материала. Однако получаемые изделия все же сохранили ряд принципиальных недостатков, к числу которых относится низкая термическая стабильность структуры [10, 12-15]. 
Примеси и легирующие элементы повышают температуру начала рекристаллизации меди даже при крайне низкой концентрации легирующего элемента. Однако применение для легирования элементов, образующих твердые растворы с высокой концентрацией, например, олова, не является эффективным в задачах повышения термической стабильности структуры [16]. Поэтому считается [17-26], что легирование элементами, имеющими наименьшую растворимость в меди ( $\mathrm{Zr}$, Hf и др.), позволяет достигнуть максимально высокой температуры начала рекристаллизации. При этом увеличение их концентрации до более чем $0,05 \%$, уже не всегда приводит к заметному росту температуры начала рекристаллизации $[17,18]$.

Задачей работы было исследовать гафниевую бронзу с минимальным содержанием в твердом растворе легирующего элемента, наноструктурированную интенсивной пластической деформацией с целью определения формирующейся при деформации структуры и уровня прочностных характеристик.

\section{2. Материал и методика исследования}

Для исследований использовалась гафниевая бронза, химический состав которой приведен в табл. 1.

Таблица 1 - Химический состав гафниевой бронзы, вес. \%

\begin{tabular}{|c|c|c|c|c|c|c|}
\hline Элемент & $\mathrm{Hf}$ & $\mathrm{O}$ & $\mathrm{Fe}$ & $\mathrm{Ni}$ & $\mathrm{Zn}$ & $\mathrm{Sn}$ \\
\hline Содержание, мас. \% & 0,78 & $\sim 0,28$ & $\sim 0,001$ & $\sim 0,005$ & $\sim 0,005$ & $\sim 0,005$ \\
\hline
\end{tabular}

Гафниевая бронза после отливки подвергалась горячей ковке при $600{ }^{\circ} \mathrm{C}$. После ковки проводилась механическая обработка и гомогенизирующий отжиг при температуре $800{ }^{\circ} \mathrm{C}$ в течение 1 ч с дальнейшим охлаждением с печью. Для этого использовали вакуумную трубчатую печь, позволяющую проводить отжиги в условиях динамического вакуума при давлении $10^{-2}-10^{-3}$ Па.

Деформирование проводили в открытых наковальнях Бриджмена диаметром 10 мм при комнатной температуре на 1, 3 и 5 об., при давлении 6 ГПа с угловой скоростью 0,3 об./мин.

Истинную деформацию $e$ рассчитывали как сумму истинной деформации сдвигом и

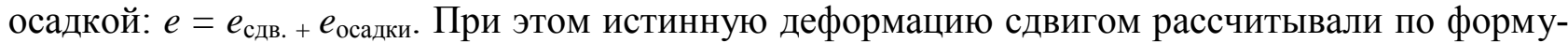
ле: $e_{\text {сдв. }}=\ln \left(1+\lambda^{2}\right)^{1 / 2}$, где $\lambda=(\varphi R) / h ; \lambda$ - деформация сдвигом при кручении; $\varphi-$ угол скручивания в радианах; $h$ - толщина образца (мм); $R$ - расстояние от оси вращения (мм). Истинную деформацию осадкой рассчитывали как $e_{\text {осадки }}=\ln \left(h_{0} / h_{\mathrm{k}}\right)$, где $h_{0}$ и $h_{\mathrm{k}}$ - толщина образца до и после деформации.

Структуру деформированных и отожженных образцов изучали с помощью просвечивающей электронной микроскопии в электронных микроскопах Philips-CM30 SuperTwin и JEM-200CX.

Измерение микротвердости материала после динамического сжатия проводили на приставке к микроскопу Neophot-21 при нагрузке, эквивалентной 40 г, по методике, описанной в работе [27].

\section{3. Результаты исследования и их обсуждение}

Микроструктура гафниевой бронзы после ковки и гомогенизирующего отжига при температуре $800{ }^{\circ} \mathrm{C}$ в течение 1 ч представлена на рис. 1. Бронза в отожженном состоянии имеет поликристаллическую структуру, с размерами кристаллитов 20-40 мкм, что значительно меньше, чем в случае чистой меди и оловянистой бронзы [16]. Кроме того, в структуре наблюдались частицы второй фазы, которые хорошо видны на нетравленом шлифе (рис. 1 б). Их размеры составляют 1-4 мкм, что согласуется с результатами, полученными в работе [18], 
в которой изучалась гафниевая бронза с содержанием 0,9 масс. \% Hf, и в исходном отожженном состоянии наблюдались частицы фазы $\mathrm{Cu}_{5} \mathrm{Hf}$ размером $\sim 3$ мкм. Поэтому, исходя из диаграммы состояния, можно ожидать, что после такой обработки большая часть гафния связана в интерметаллиды $\mathrm{Cu}_{5} \mathrm{Hf}$. После травления выявляется зеренная структура (рис. 1 a). Зерна имеют размеры от 20 до 100 мкм. В некоторых участках в небольшом количестве присутствуют двойники отжига. Микротвердость в исходном отожженном состоянии составляла $760 \pm 20$ МПа.

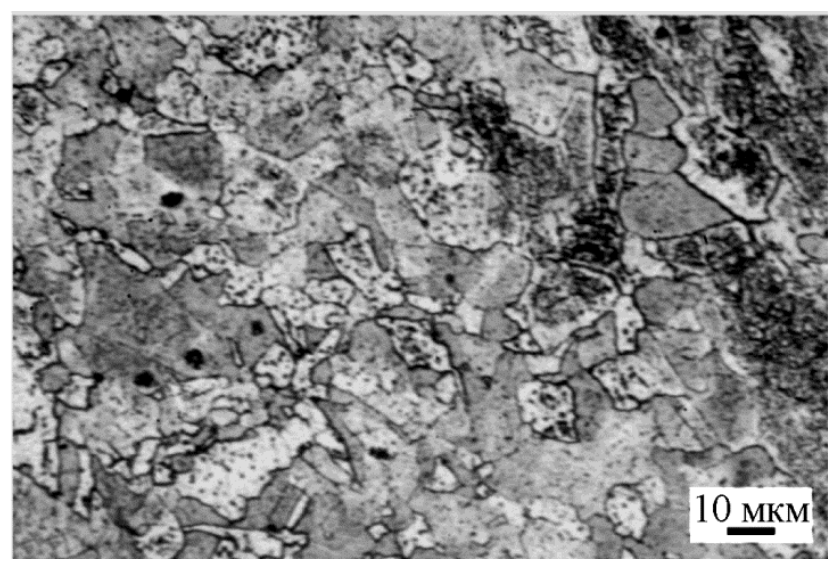

$a$

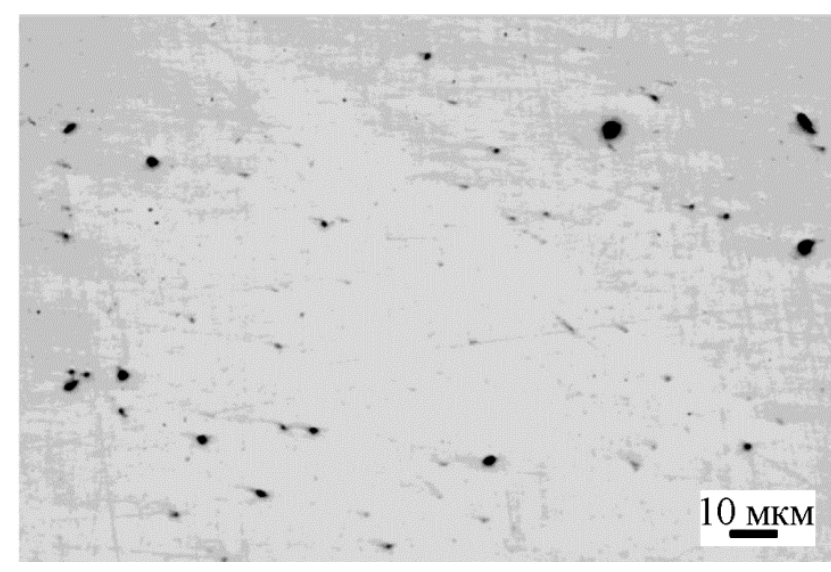

$\sigma$

Рис. 1. Микрофотографии структуры гафниевой бронзы после ковки и отжига при температуре $800{ }^{\circ} \mathrm{C}$ в течение 1 ч: $a$ - травленый; $\sigma$ - нетравленый шлиф

Необходимо отметить, что расчетная величина деформации при КВД зависит от расстояния от оси вращения и деформация распределена неравномерно по радиусу образца, увеличиваясь от центра к периферии. Соответственно структура измельчается неравномерно, особенно при малых величинах деформации (количестве оборотов), и самая мелкая структура формируется в периферийных областях деформируемых дисков. Поэтому для корректного сравнения структуры после разной обработки (например после разного числа оборотов), как правило, рассматривают структуру на середине радиуса образцов. Оценочные величины истинной деформации на середине радиуса образцов представлены в табл. 2.

Таблица 2 - Расчетная величина истинной деформации, достигаемой при деформировании методом КВД на середине радиуса образцов

\begin{tabular}{|c|c|c|c|}
\hline Обороты КВД & 1 & 3 & 5 \\
\hline$e$ & $\sim 4,6$ & $\sim 5,9$ & $\sim 6,5$ \\
\hline
\end{tabular}

Проведенные исследования показали, что после одного оборота КВД в гафниевой бронзе формируется градиентная по радиусу диска дисперсная субмикрокристаллическая структура с преимущественно высокоугловыми разориентировками между кристаллитами. В центральной части образца, где степень деформации минимальна и структура наименее фрагментирована, средний размер кристаллитов составляет 200 нм (рис. $2 a, \sigma$ ). На снимках виден специфический контраст внутри кристаллитов, указывающий на высокий уровень внутренних упругих напряжений. Границы некоторых кристаллитов малоугловые, напоминающие дислокационные скопления, хотя имеются и ровные тонкие высокоугловые границы. При этом на некоторых электронограммах видны близко расположенные рефлексы, образующие радиально вытянутые скопления на Дебаевских кольцах, и их количество невелико. Такие электронограммы можно охарактеризовать как переходные от точечных, характер- 
ных для крупнокристаллической структуры, к кольцевым, которые указывают на образование субмикрокристаллической структуры.

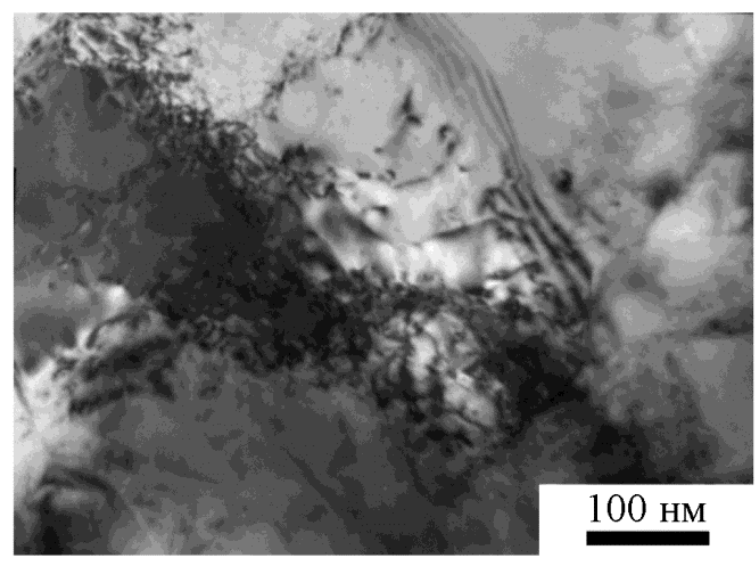

$a$

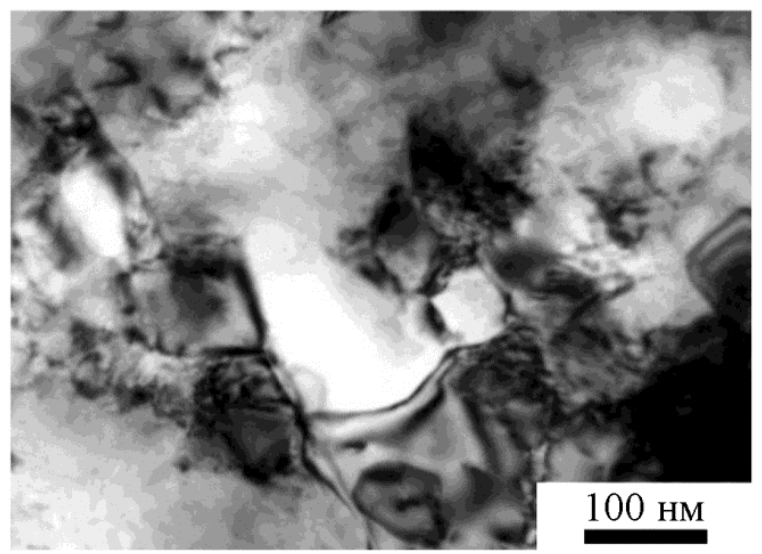

B

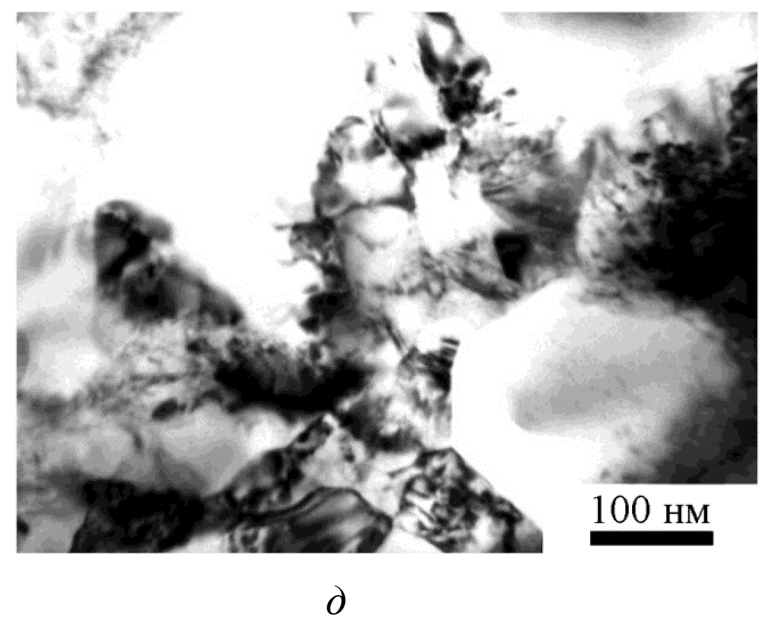

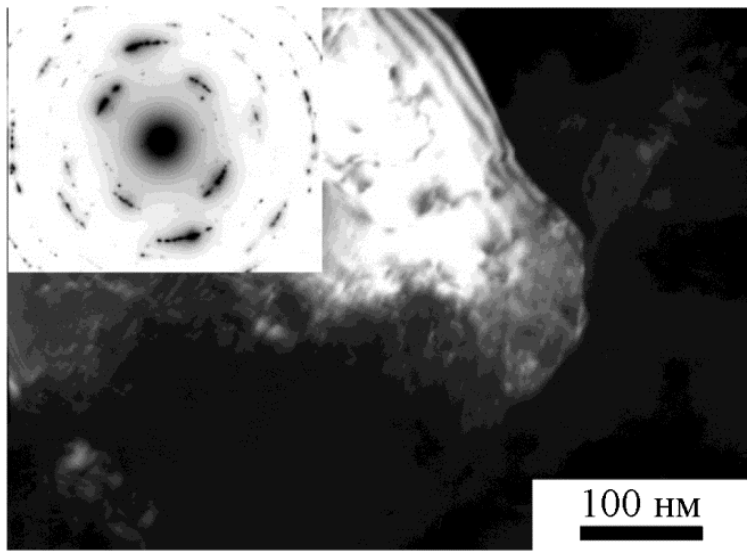

$\sigma$

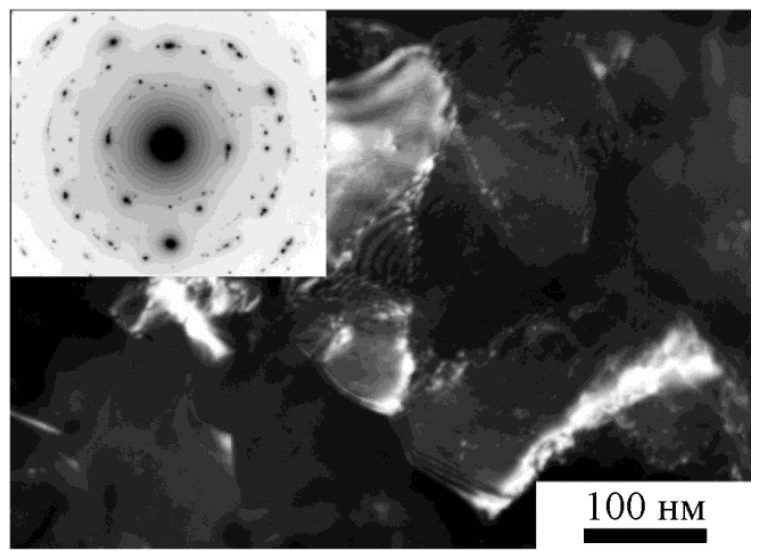

2

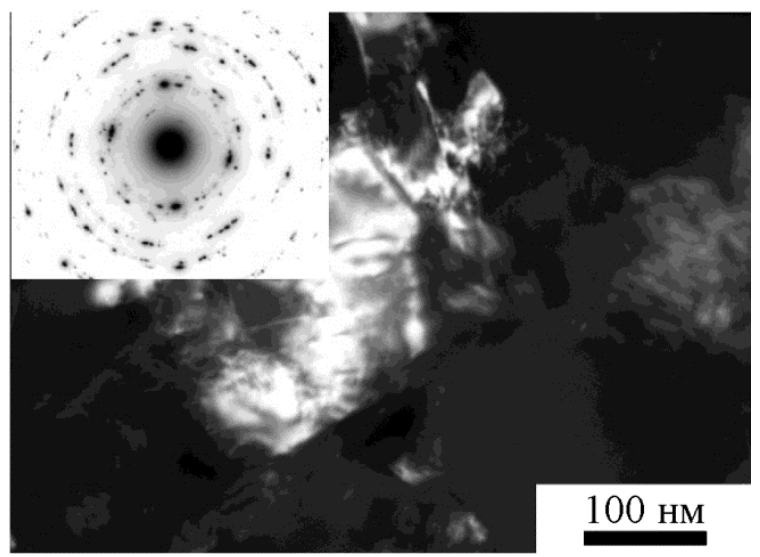

$e$

Рис. 2. Электронные микрофотографии структуры и электронограммы в центральной части образца гафниевой бронзы $(a, \sigma)$, на середине радиуса $(b, 2)$ и в периферийной части $(\partial, e)$ после КВД на 1 об.: $(a, b, \partial)$ - светлые поля; $(\sigma, 2, e)$ - темные поля; вставки - электронограммы

С увеличением деформации при удалении от центра структура становится более дисперсной и средний размер кристаллитов уменьшается до 180 нм (рис. 2 в, г). Поскольку в данной области образца деформации выше, то и уровень внутренних упругих напряжений 
значительно выше, и это видно по увеличению плотности дислокаций. Также видно, что электронограммы, в основном кольцевые, с уже достаточно большим количеством рефлексов на Дебаевских кольцах и их расположение более равномерно (рис. 2 г).

Наличие различной структуры в зависимости от радиуса указывает на ее зависимость от градиента деформации, характерного для метода КВД. При этом градиентный характер структуры подтверждается изменением микротвердости по радиусу образца. Значения микротвердости составляют $1740 \pm 60$ МПа в центре образца, $1820 \pm 20$ МПа на середине радиуса и $2050 \pm 40$ МПа на краю образца. При этом с увеличением деформации (числа оборотов) КВД ожидаемо происходит как повышение микротвердости, так и ее выравнивание по радиусу образца (рис. 3). Такое выравнивание микротвердости с ростом величины деформации вполне ожидаемо, однако данный процесс не происходит в полной мере, что видно по изменению микротвердости для 5 об. КВД. Это указывает на сохраняющуюся зависимость микроструктуры от положения участка в плане его удаленности от центра.

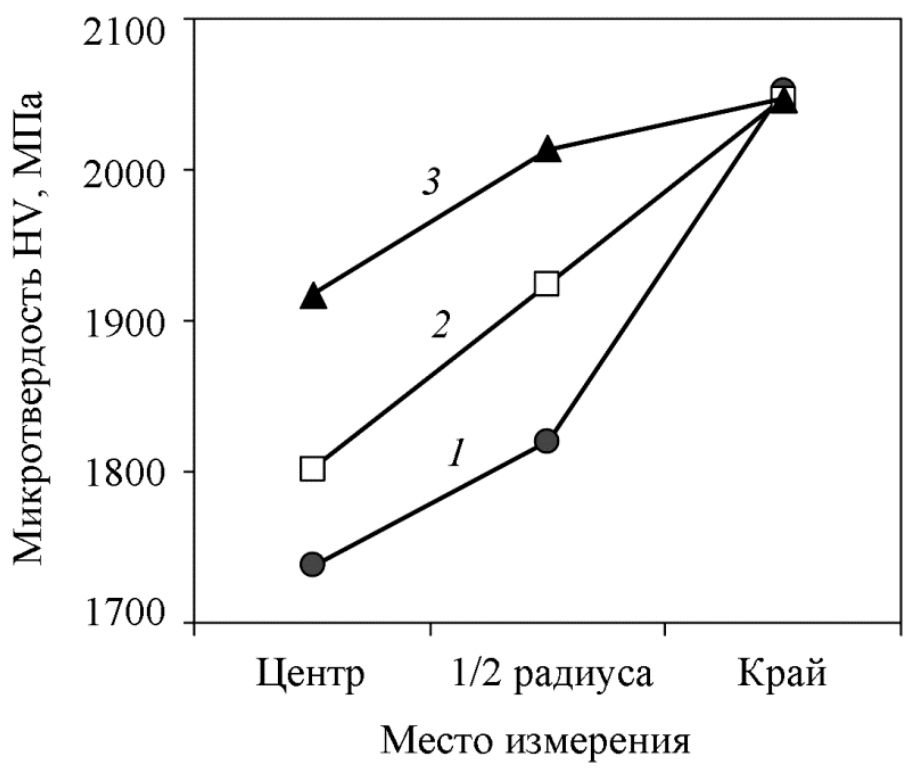

Рис. 3. Изменение микротвердости гафниевой бронзы по радиусу образцов после деформирования методом КВД с различным числом оборотов:

$$
1 \text { - } 1 \text { об.; } 2 \text { - } 3 \text { об.; } 3 \text { - } 5 \text { об.; }
$$

Поскольку в бронзе после КВД на 3 и 5 об. микротвердость становится более равномерной по радиусу образцов с увеличением деформации, то можно было ожидать и более однородной структуры.

Действительно, после 3 об. КВД структура становится более однородной, однако различия по радиусу все же присутствуют (рис. 4). Структура заметно измельчилась по сравнению с представленной выше структурой после 1 об. КВД для снимков с середины радиуса образца. Средний размер кристаллитов в этом состоянии составляет 150 нм. Границы кристаллитов имеют искривления, а внутри кристаллитов наблюдается характерный для напряженного состояния контраст, что косвенно свидетельствует о высоком уровне внутренних напряжений и особом «неравновесном» состоянии границ. Однако практически на всех электронограммах Дебаевские кольца все еще не сформированы достаточным количеством рефлексов (рис. 4 б).

Поскольку в периферийной части образцов для 1, 3 и 5 об. в микротвердость повышается до практически одного и того же значения $2050 \pm 40$ МПа, то можно было ожидать близкой по параметрам микроструктуры (рис. 3). Структура в этой части образца для 5 об. показана на рис. 4 в, г и в целом очень похожа на структуру после 1 об. (рис. $2 \partial, e$ ). 


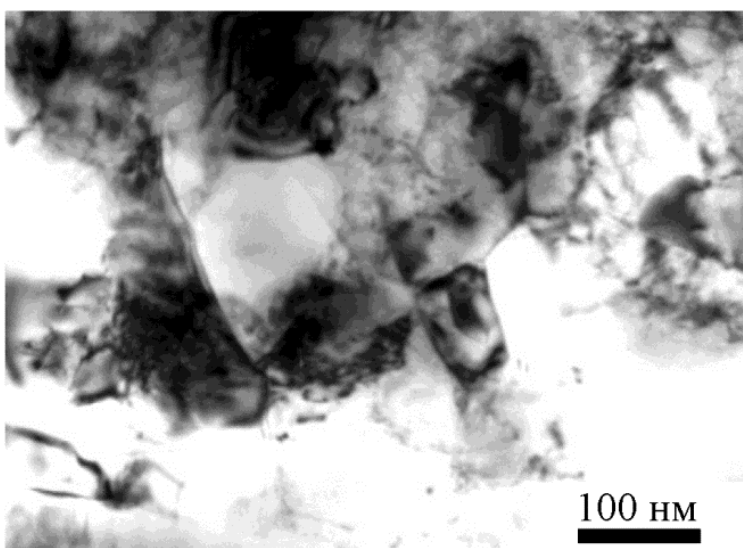

$a$

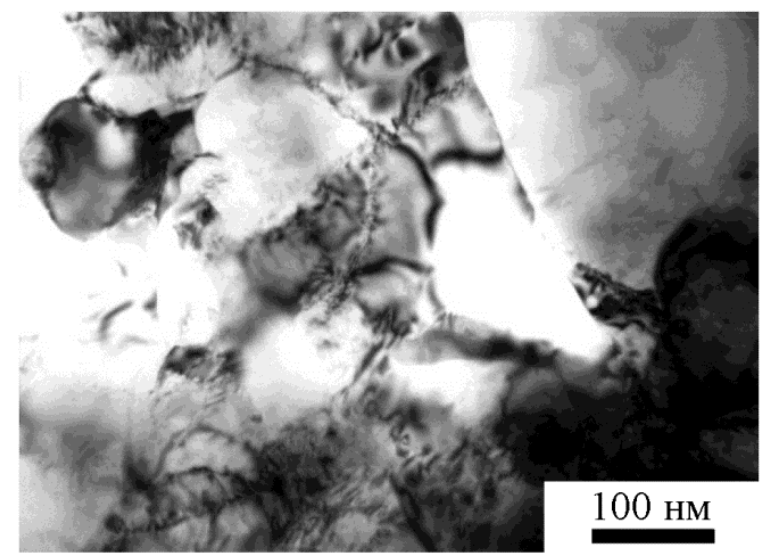

B

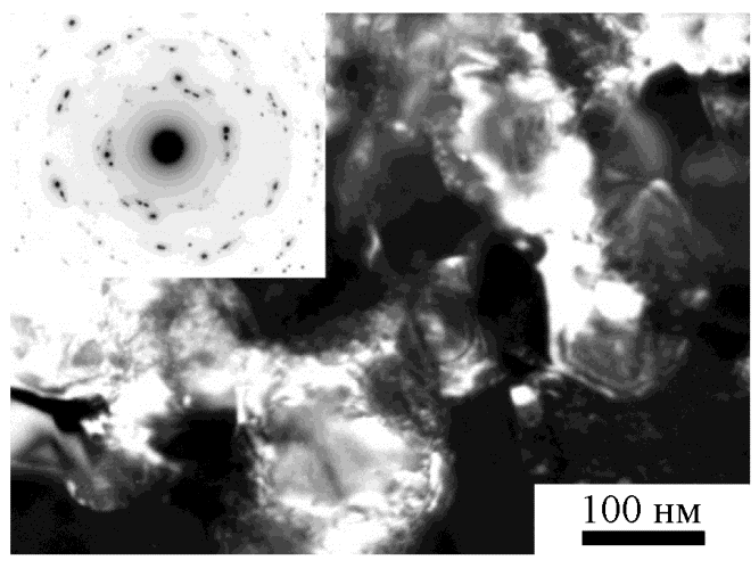

$\sigma$

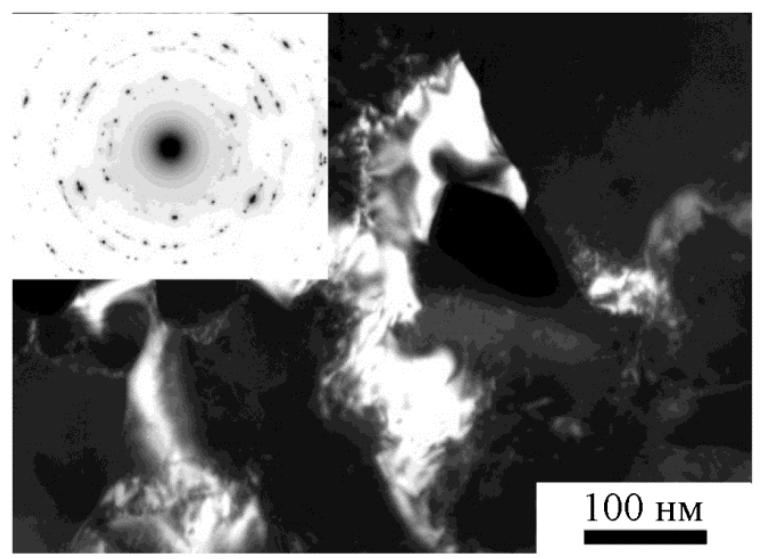

2

Рис. 4. Электронные микрофотографии структуры и электронограммы в центральной части образца гафниевой бронзы после КВД на 3 об. а $(a, \sigma)$ и в периферийной части $(b, 2)$ после

КВД на 5 об.: $(a, b)$ - светлые поля; $(\sigma, 2)$ - темные поля; вставки - электронограммы

Средний размер кристаллитов, достигаемый при КВД 5 об. в периферийной части, составляет приблизительно 120 нм. Однако структура все еще неоднородна и на Дебаевских кольцах электронограмм еще не наблюдается равномерно распределенных рефлексов. В то же время на снимках с периферийной части образцов появляются области с элементами структуры, претерпевшей процессы релаксации. Есть зерна с прямыми ровными границами, но не равноосной, а вытянутой формы, а внутри кристаллитов наблюдается характерный для напряженного состояния контраст. Наблюдается и значительный локальный разброс кристаллитов по размерам. Все это указывает на протекание релаксационных процессов непосредственно при деформировании и может служить ограничением для дальнейшего измельчения зерен и повышения микротвердости данного материала.

Более наглядно упрочнение с ростом деформации гафниевой бронзы демонстрирует рис. 5, на котором значения микротвердости нанесены в зависимости от числа оборотов КВД при комнатной температуре. 


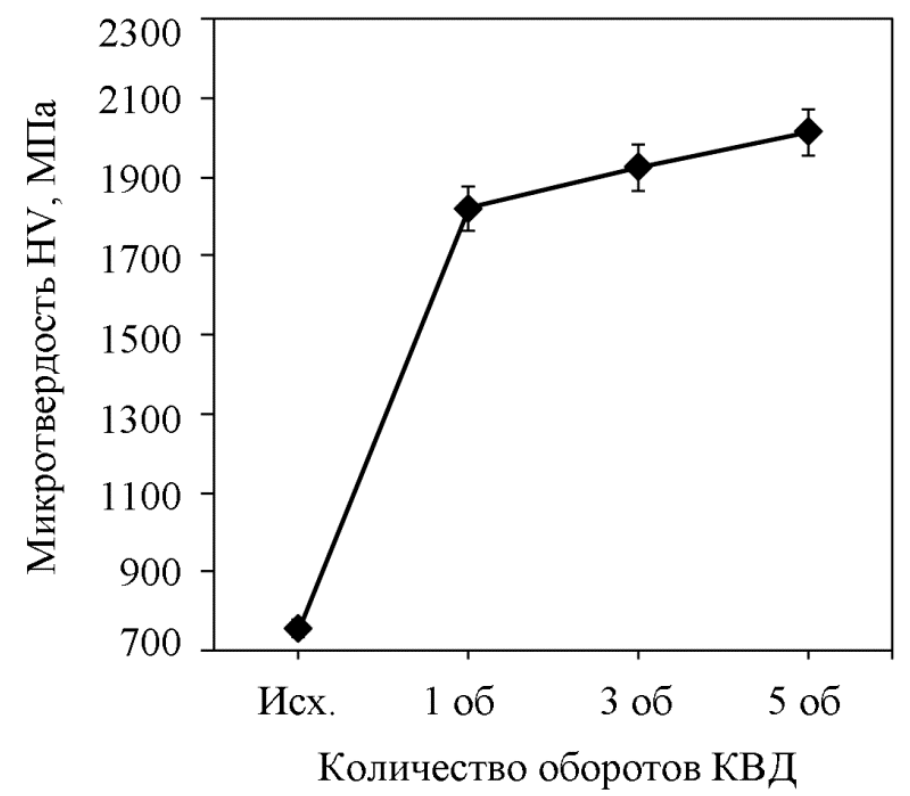

Рис. 5. Изменение микротвердости гафниевой бронзы при КВД в зависимости от числа оборотов (на середине радиуса образцов)

Микротвердость при КВД постепенно возрастает, однако изменения не столь значительны, как в оловянистой бронзе [28], и являются неким средним состоянием между значениями, полученными для оловянистой бронзы и меди технической чистоты стандарта M1 [14]. Так, для гафниевой бронзы характерно значительное упрочнение уже при одном обороте КВД, как и для оловянистой бронзы. Однако с дальнейшим увеличением деформации в структуре наблюдаются характерные элементы, указывающие на прохождение релаксационных процессов, как наблюдалось при исследовании меди, и рост микротвердости замедляется. При этом полученные структуры гафниевой бронзы стабильны при комнатной температуре, как и у образцов оловянистой бронзы [28], тогда как для медных образцов наблюдалось изменение структуры после снятия нагрузки при вылеживании деформированных образцов при комнатной температуре [14].

Можно заключить, что применение кручения под высоким давлением позволяет значительно сильнее измельчать зеренную структуру образцов из гафниевой бронзы уже при 1 об. КВД, в сравнении с методом РКУП [19, 23], вследствие большей деформации, несмотря на градиентное изменение структуры по радиусу образцов. Также в образцах, подвергнутых КВД, в целом структура не только более дисперсная, но и более равномерная с преобладанием равноосных кристаллитов, по сравнению со структурой после РКУП, в которой явно выражены полосы деформации и протяженные двойники [19, 23].

\section{4. Заключение}

Проведенные исследования показали, что даже в случае с минимальным содержанием в твердом растворе гафния, структура всех исследованных образцов бронзы после КВД является стабильной при комнатной температуре и не претерпевает каких-либо изменений после снятия нагрузки и длительном вылеживании.

При этом уже после одного оборота КВД в гафниевой бронзе формируется градиентная по радиусу диска дисперсная субмикрокристаллическая структура со средним размером кристаллитов около 200 нм и преимущественно высокоугловыми разориентировками между кристаллитами. С увеличением деформации при удалении от центра структура становится более дисперсной и средний размер кристаллитов уменьшается до 180 нм. А средний размер кристаллитов, достигаемый при КВД 5 об. в периферийной части, составляет прибли- 
зительно 120 нм. Однако структура все еще неоднородна, что подтверждается измерениями микротвердости, и наблюдается значительный локальный разброс кристаллитов по размерам.

В то же время полученное при гомогенизирующем отжиге содержание гафния, повидимому, уже не обеспечивает условий для достижения однородной нанокристаллической структуры даже при 5 об. КВД из-за протекания релаксационных процессов непосредственно при деформировании, что и является ограничением для измельчения зерна и повышения микротвердости при данной обработке.

\section{Благодарности}

Электронно-микроскопическое исследование выполнено на оборудовании ичентра коллективного пользования в Испытательном иентре нанотехнологий и перспективных материалов ИФМ УрО РАН.

Работа выполнена в рамках государственного задания ФАНО России (тема «Функичия номер госрегистраџии АAАA-A19-119012990095-0) при частичной поддержке программы фундаментальных исследований УрО РАН (проект 18-10-2-37).

\section{Литература}

1. Valiev R. Z., Zhilyaev A. P., Langdon T. G. Bulk Nanostructured Materials: Fundamentals and Applications. - New Jersey : TMS, Wiley, Hoboken, 2014. - 440 p. - ISBN 978-1-118-09540-9.

2. Gleiter H. Nanostructured materials: basic concepts and microstructure // Acta Mater. 2000. - Vol. 48. - P. 1-29. - DOI: 10.1016/S1359-6454(99)00285-2.

3. Nanocrystalline grain structures developed in commercial purity $\mathrm{Cu}$ by low temperature cold rolling / Y. M. Wang, M. W. Chen, H. W. Sheng, E. Ma // Mater. Res. Soc. - 2002. - Vol. 17, no. 12. - P. 3004-3007. - DOI: 10.1557/JMR.2002.0436.

4. On the structure and strength of ultra-fine-grained copper produced by severe plastic deformation / V. Y. Gertsman, R. Birringer, R. Z. Valiev, H. Gleiter // Scr. Met. \& Mater. - 1994. Vol. 30, no. 2. - P. 229-234. - DOI: 10.1016/0956-716X(94)90045-0.

5. Erbel S. Mechanical properties and structure of extremely strainhardened copper // Metals Technol. - 1979. - Vol. 6, no. 1. - P. 482-486. - DOI: 10.1179/030716979803276363.

6. Liao X. Z., Zhao Y. H., Zhu Y. T. Grain-size effect on the deformation mechanisms of nanostructured copper processed by high-pressure torsion // J. Appl. Phys. - 2004. - Vol. 96, no. 1. - P. 636-640. - DOI: doi.org/10.1063/1.1757035.

7. Microstructures and properties of copper processed by equal channel angular extrusion for 1-16 passes / F. Dalla Torre, R. Lapovok, J. Sandlin, P. F. Thomson, C. H. J. Davies, E. V. Pereloma // Acta Mater. - 2004. - Vol. 52, no. 16. - P. 4819-4832. - DOI: 10.1016/j.actamat.2004.06.040.

8. Deformation behavior of ultra-fine-grained copper / R. Z. Valiev, E. V. Kozlov, Yu. F. Ivanov, J. Lian, A. A. Nazarov, B. Baudel // Acta Met. \& Mater. - 1994. - Vol. 42, no. 7. P. 2467-2475. - DOI: 10.1016/0956-7151(94)90326-3.

9. Alexandrov I. V., Dubravina A. A., Kim H. S. Nanostructure formation in copper subjected to high pressure torsion // Defect \& Diffus. Forum. - 2002. - Vol. 208-209. - P. 229-232. DOI: 10.4028/www.scientific.net/DDF.208-209.229.

10. Grain coarsening during compression of bulk nanocrystalline nickel and copper / S. Brandstetter, K. Zhang, A. Escuadro, Julia Weertman // Scr. Mater. - 2008. - Vol. 58, no. 1. P. 61-64. - DOI: 10.1016/j.scriptamat.2007.08.042.

11. Horita Z., Langdon T. G. Microstructures and microhardness of an aluminum alloy and pure copper after processing by high-pressure torsion // Mater. Sci. \& Eng. A. - 2005. - Vol. 410-411. P. 422-425. - DOI: 10.1016/j.scriptamat.2007.08.042. 
12. Islamgaliev R. K., Chmelik F., Kuzel R. Thermal stability of submicron grained copper and nickel // Mater. Sci. \& Eng. A. - 1997. - Vol. 237, no. 1. - P. 43-51. - DOI: 10.1016/S09215093(97)00107-X.

13. Akhmadeev N. H., Kobelev N. P., Mulyukov R. R. The effect of heat treatment on the elastic and dissipative properties of copper with the submicrocrystalline structure // Acta Met. \& Mater. 1993. - Vol. 41, no 4. - P. 1041-1046. - DOI: 10.1016/0956-7151(93)90153-J.

14. Structure and Thermal Stability of $\mathrm{Cu}$ after Severe Plastic Deformation / V. V. Popov, A. V. Stolbovskiy, E. N. Popova, V. P. Pilyugin // Defect and Diffusion Forum. - 2010. Vol. 297-301. - P. 1312-1321. - DOI: 10.4028/www.scientific.net/DDF.297-301.1312.

15. Structure, thermal stability, and state of grain boundaries of copper subjected to highpressure torsion at cryogenic temperatures / A. V. Stolbovsky, V. V. Popov, E. N. Popova, V. P. Pilyugin // Bulletin of the Russian Academy of Sciences: Physics. - 2014. - Vol. 78. P. 908-916. - DOI: 10.3103/S1062873814090299.

16. Effect of severe plastic deformation by high-pressure torsion on the structure and properties of copper and tin bronze / A. V. Stolbovsky, V. V. Popov, E. N. Popova, R. M. Falakhutdinov // Diagnostics, Resource and Mechanics of materials and structures. - 2017. - Iss. 5. - P. 13-22. DOI: 10.17804/2410-9908.2017.5.013-022. - URL: http://dream-journal.org/issues/2017-5/20175_144.html (accessed: 19.02.2021)

17. Shangina D. V., Bochvar N. R., Dobatkin S. V. Structure and properties of ultrafine-grained $\mathrm{Cu}-\mathrm{Cr}$ alloys after high pressure torsion // Materials Science Forum. - 2011. - Vol. 667-669. P. 301-306. - DOI: 10.4028/www.scientific.net/MSF.667-669.301.

18. Shangina D. V., Bochvar N. R., Dobatkin S. V. The effect of alloying with hafnium on the thermal stability of chromium bronze after severe plastic deformation // Journal of Materials Science. - 2012. - Vol. 47. - P. 7764-7769. - DOI: 10.1007/s10853-012-6525-9.

19. Structure and properties of $\mathrm{Cu}$ alloys alloying with $\mathrm{Cr}$ and $\mathrm{Hf}$ after equal channel angular pressing / D. V. Shangina, Yu. M. Maksimenkova, N. R. Bochvar, Vl. Serebryany, G. Raab, A. Vinogradov, Werner Skrotzki, S. Dobatkin // Advanced Materials Research. - 2014. - Vol. 922. P. 651-656. - DOI: 10.4028/www.scientific.net/AMR.922.651.

20. Effect of deformation schedules and initial states on structure and properties of $\mathrm{Cu}-0.18 \% \mathrm{Zr}$ alloy after high-pressure torsion and heating / S. V. Dobatkin, D. V. Shangina, N. R. Bochvar, Miloš Janečekc // Materials Science and Engineering A. - 2014. - Vol. 598. - P. 288-292. DOI: 10.1016/j.msea.2013.12.104.

21. Improvement of strength and conductivity in $\mathrm{Cu}$-alloys with the application of highpressure torsion and subsequent heat-treatments / D. V. Shangina, J. Gubicza, E. Dodony, N. R. Bochvar, P. B. Straumal, N. Yu. Tabachkova \& S. V. Dobatkin // Journal of Materials Science. - 2014. - Vol. 49. - P. 6674-6681. - DOI: 10.1007/s10853-014-8339-4.

22. High strength and good electrical conductivity in $\mathrm{Cu}-\mathrm{Cr}$ alloys processed by severe plastic deformation / S. V. Dobatkin, J. Gubicza, D. V. Shangina, N. R. Bochvara, N. Y. Tabachkova // Materials Letters. - 2015. - Vol. 153. - P. 5-9. - DOI: doi.org/10.1016/j.matlet.2015.03.144.

23. Dobatkin S. V., Bochvar N. R., Shangina D. V. Aging processes in ultrafine-grained lowalloyed bronzes subjected to equal channel angular pressing // Advanced Engineering Materials. 2015. - Vol. 17. - P. 1862-1868. - DOI: 10.1002/adem.201500099.

24. Influence of microalloying with zirconium on the structure and properties of $\mathrm{Cu}-\mathrm{Cr}$ alloy after high pressure torsion / D. V. Shangina, N. R. Bochvar, M. V. Gorshenkov, H. Yanar, G. Purcek, S. V. Dobatkin // Materials Science and Engineering A. - 2016. - Vol. 650. - P. 63-66. DOI: $10.1016 /$ j.msea.2015.10.008.

25. Optimization of strength, ductility and electrical conductivity of $\mathrm{Cu}-\mathrm{Cr}-\mathrm{Zr}$ alloy by combining multi-route ECAP and aging / G. Purcek, H. Yanar, M. Demirtas, Y. Alemdag, D. V. Shangina, S. V. Dobatkin // Materials Science and Engineering A. - 2016. - Vol. 649. - P. 114-122. DOI: 10.1016/j.msea.2015.09.111. 
26. Influence of alloying with hafnium on the microstructure, texture, and properties of $\mathrm{Cu}-\mathrm{Cr}$ alloy after equal channel angular pressing / D. Shangina, Yu. Maksimenkova, N. Bochvar, Vl. Serebryany, Georgy Raab, A. Vinogradov, Werner Skrotzki \& S. Dobatkin // Journal of Materials Science. - 2016. - Vol. 51. - P. 5493- 5501. - DOI: 10.1007/s10853-016-9854-2.

27. Stolbovsky A. V., Popov V. V., Popova E. N. Structure and Thermal Stability of Tin Bronze Nanostructured by High Pressure Torsion // Diagnostics, Resource and Mechanics of materials and structures. - 2015. - Iss. 5. - P. 118-132. - DOI: 10.17804/2410-9908.2015.5.118-132. URL: http://dream-journal.org/issues/2015-5/2015-5_52.html (accessed: 27.06.2020).

28. Evolution of the Structure of $\mathrm{Cu}-1 \%$ Sn Bronze under High Pressure Torsion and Subsequent Annealing / V. V. Popov, E. N. Popova, A. V. Stolbovsky, R. M. Falahutdinov // Physics of Metals and Metallography. - 2018. - Vol. 119. - P. 358-367. - DOI: 10.1134/S0031918X18040154. 